\title{
Temporal perturbations of binocular rivalry
}

\author{
RANDOLPH BLAKE \\ Vanderbilt University, Nashville, Tennessee \\ DAVID WESTENDORF \\ University of Arkansas, Fayetteville, Arkansas \\ and \\ ROBERT FOX \\ Vanderbilt University, Nashville, Tennessee
}

\begin{abstract}
Successive durations of binocular rivalry are sequentially independent, random variables. To explore the underlying control process, we perturbed the cycle during a 30-sec viewing period by immediately forcing an eye to return to dominance whenever it became suppressed. During this period of forced dominance, that eye's individual dominance durations were unusually brief, but immediately following the period of forced dominance that eye's suppression durations were unusually long. However, no long-term change in the sequential pattern of rivalry occurred, and the stochastic independence of successive durations was maintained during and following the period of forced dominance. The same pattern of results was obtained with even longer periods of forced dominance. These results are consistent with the existence of a short-term adaptation, or fatigue, process responsible for transitions from dominance to suppression.
\end{abstract}

Among the fascinating features of binocular rivalry are the abrupt, seemingly unpredictable shifts in dominance and suppression that occur over time. Casual observation suggests that these shifts are unrelated in any compelling way to volitional attempts to alter the temporal course of rivalry alternations, and this suggestion is borne out by results from experiments in which observers tried but largely failed to maintain dominance of one eye's view indefinitely (Lack, 1978). Moreover, this phenomenal impression of unpredictability receives quantitative support from stochastic analyses, which reveal that successive durations of phases of dominance and suppression are sequentially independent random variables (Fox \& Herrmann, 1967; Wade, 1975; Walker, 1975). The duration of any given phase, in other words, is unrelated to the duration of prior phases.

These conclusions are based on data gathered by voluntary reports of practiced observers who have viewed rivalry for intervals on the order of $1 \mathrm{~min}$, with interspersed periods of rest. The stimulus conditions were designed to promote abrupt, clear-cut changes in phenomenal state, with minimum incidence of intermediate, or mixed, dominance. It is unlikely, however, that these conclusions are peculiar either to these stimulus conditions or to the response tendencies of observers, for the same pattern of sequential independence is found when

This work was supported by National Institutes of Health Grants EY07760 and EY00590. Portions of this work were described at the 1989 meetings of the Psychonomic Society in Atlanta, GA. Correspondence should be addressed to Randolph Blake, Department of Psychology, Vanderbilt University, Nashville, TN 37240. the changes in phenomenal states are signaled by optokinetic nystagmus, an involuntary, objective indicator of rivalry fluctuations (Fox, Todd, \& Bettinger, 1975). Randomness appears, then, to be an inherent property of the rivalry mechanism itself.

The discovery of any random process leads naturally to questions about its source. In the case of rivalry, it is reasonable to hypothesize that peripheral visual variables constitute the source of randomness. For instance, Levelt (1965) proposed that a requisite number of microsaccadic eye movements trigger an eye's transition from suppression to dominance. That specific hypothesis, however, as well as others predicated on the operation of peripheral variables such as shifts in accommodation or in pupil size are invalidated by stochastic analyses of rivalry fluctuations produced by afterimages (Blake, Fox, \& McIntyre, 1971; Wade, 1975). The temporal pattern obtained under these conditions is identical to those observed with conventional rival stimuli. Taken together, then, investigations of the time course of binocular rivalry alternations make it clear that random variation is an intrinsic component of some central mechanism, and that this randomness must be incorporated within any comprehensive theoretical account of binocular rivalry.

Besides dealing with randomness, any successful account of rivalry must deal with the process responsible for alternations in monocular dominance over time. It is not immediately obvious why an eye once dominant in rivalry succumbs so quickly to the other eye. Why, in other words, is vision unstable during rivalry? Several recent theories of rivalry have instantiated the classic concept of reciprocal inhibition within a neural network (see, 
e.g., Lehky, 1988). These models incorporate the notion of fatigue, or adaptation, during dominance, which steadily weakens the dominant eye's excitatory drive to the point at which dominance can no longer be sustained. Once suppressed, however, that eye begins to recover from adaptation. This notion of fatigue during dominance and recovery during suppression leads to predictions that have heretofore been untested.

In the present experiment, we have studied the alternation process, including its randomness, by examining the temporal sequence of rivalry alternations under conditions in which the natural switch between phenomenal states was forestalled. Specifically, we forced a given eye to remain dominant by immediately returning that eye to dominance whenever it became suppressed. Forced dominance was accomplished by introducing an abrupt transient in that eye's rival target whenever the observer declared that the target had become suppressed-a maneuver that effectively restored the suppressed target to dominance. The temporal consequences of this manipulation were analyzed both during the period of forced dominance and for a short period following forced dominance, when rivalry was aliowed to return to its freerunning mode. As will be discussed in the last section of this paper, results from this analysis bear on the nature of the process responsible for rivalry alternations.

\section{METHOD}

\section{Visual Displays}

Dichoptic displays that engage in binocular rivalry were generated on a pair of gray-scale video monitors (12-in. Apple monitors; $66.7-\mathrm{Hz}$ frame rate; $\mathrm{P} 4$ phosphor; $640 \times 420$ pixel resolution) under the control of a Macintosh II computer. The targets, shown in Figure 1A, consisted of a central disk surrounded by an annulus, which itself was surrounded by a square frame. In one eye's view, the disk was black, while in the other eye's view the disk was white; in both eyes' views, the disks were surrounded by a white inner annulus and a black outer annulus. The angular dimensions of the various components of these displays are given in Figure 1A. The luminance of the black portions of the display was $0.07 \mathrm{fL}$, the luminance of the white portions was $26 \mathrm{fL}$ ( $1.0 \mathrm{fL}$ for DW-dim), and the luminance of the gray surround was $13 \mathrm{fL}$. In these experiments, the black disk was always presented to the left eye and the white disk to the right eye.

The observer viewed the two video monitors (and hence the two rival targets) through a mirror stereoscope, such that each eye saw a separate monitor. The gray square serving as the background for each display provided a strong stimulus for binocular alignment of the two targets. Great care was taken in adjusting the mirrors of the stereoscope to ensure proper binocular alignment of the displays for each observer.

\section{Procedure}

Observers tracked alternations in dominance between the two targets, using a pair of keys on the computer keyboard. The instructions were to depress one key when the black disk was completely dominant, with no trace of the white disk, and to depress the other key when the white disk was dominant exclusively. During periods of mixed dominance, neither key was to be depressed. In fact, the observers experienced only very brief periods of mixed dominance with these small rival targets. Dominance durations were measured by the computer to within 5 -msec accuracy.
Except where noted, data were collected in 45-sec tracking episodes, with the first $30 \mathrm{sec}$ consisting of perturbation cycles and the last $15 \mathrm{sec}$ consisting of unperturbed cycles. During the perturbation period, a given eye was forced to remain dominant by always introducing a sharp transient to that eye whenever the observer signaled suppression of that eye's target. Figure $1 \mathrm{~B}$ schematically illustrates this perturbation sequence. In the top time line, rivalry is shown alternating between the two eyes, with no perturbations introduced. The bottom time line shows a condition where the right eye stimulus is perturbed (indicated by the upward pointing arrows) every time the left eye becomes dominant. This was accomplished in the following way. Whenever the observer depressed the key signaling dominance of the black disk (hence suppression of the white disk presented to the right eye), the suppressed white disk was abruptly replaced by the black disk for a 250 -msec period, after which the white disk reappeared. This flash maneuver was repeated throughout the first $30 \mathrm{sec}$ of the tracking episode (i.e., during the perturbation period) each time the designated eye became suppressed. On trials where left-eye dominance was to be maintained, the black disk was briefly replaced with the white one whenever the observer signaled dominance of the white disk (i.e., suppression of the black disk seen by the left eye). It is well known that a brief, strong flash to a suppressed eye typically terminates suppression and returns that eye to dominance (e.g., Wolfe, 1984). In Figure 1B, this premature termination of suppression is illustrated by the brief dominance durations for the left eye; the right eye returns to dominance shortly after the perturbation demarked by the arrows. During the 15 -sec period following the perturbation period (not illustrated in Figure 1B), the rival targets remained unchanged (i.e., unperturbed). On control trials (upper portion of Figure 1B), the two targets remained unperturbed throughout the 45 -sec tracking episode. At least 10 tracking episodes were devoted to each condition (forced left-eye dominance, forced right-eye dominance, control trials), with trials delivered in a pseudorandom order and interspersed with brief rest periods.

\section{Observers}

Six individuals participated in this experiment, 2 of the authors (R.B., D.W.) and 4 who were naive about the purpose of the experiment. All had normal or corrected-to-normal vision and good stereopsis. Each was given practice on the task before data were formally collected.

\section{RESULTS}

Figure 2 summarizes all the rivalry tracking data for observer R.B. For each of the three graphs, the ordinate gives the duration of a dominance phase, and the abscissa specifies when that duration terminated during the tracking episode. These plots, in other words, lay out the temporal course of rivalry for the unperturbed (middle graph in Figure 2) and perturbed (lower graph in Figure 2) eyes during the 45 -sec tracking episode; also shown are dominance durations measured under control conditions involving no perturbations (upper graph in Figure 2). In the remainder of this section, we shall refer to this figure to illustrate various points about the results.

\section{Was Forced Dominance Achieved?}

Our experiment depends on the success of the target flash in terminating suppression, thereby forcing the return of the eye to dominance. For 5 of 6 observers, this manipulation was reliably effective-their dominance durations for the nonperturbed eye were consistently $1 \mathrm{sec}$ 

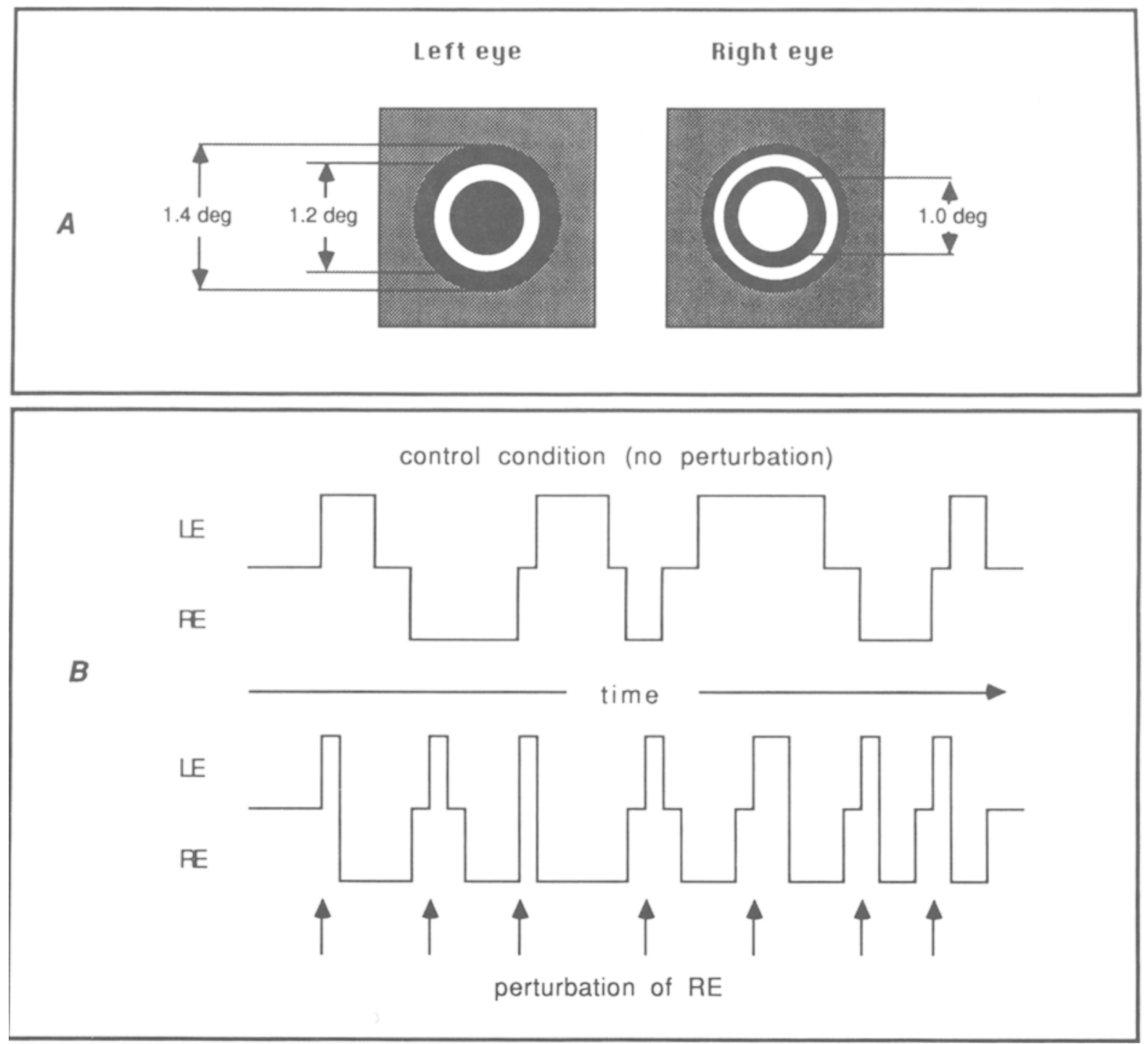

Figure 1. (A) Schematic of rival targets and their angular dimensions. (B) Hypothetical tracking sequence to illustrate the perturbation technique. $\mathrm{LE}=$ left eye. $\mathrm{RE}=$ right eye. The upper sequence (control condition) shows alternations in monocular dominance during free-running rivalry. The lower sequence (perturbation of right eye) shows that the right eye's stimulus was transiently changed at the onset of dominance of the left eye; the brief transient to the right eye is indicated by the arrows, and the brief dominance durations for the left eye signify that the previously suppressed right eye was prematurely returned to dominance (and the left eye hence returned to suppression) by virtue of the perturbation. The short horizontal lines interspersed between successive monocular dominance periods are meant to indicate those brief intervals of mixed dominance which occurred infrequently.

or less and averaged $794 \mathrm{msec}$. In the middle graph in Figure 2 (unperturbed eye), this result is shown by the clustering of dominance durations around a value slightly less than 1.0 during the initial 30 -sec perturbation period; these are individual dominance durations for the unperturbed eye (i.e., suppression durations for the perturbed eye). In Figure 1B, these abbreviated dominance durations are illustrated for the left eye. Regardless of when it occurred during the 30-sec period, the perturbation was effective in terminating suppression (note the essentially flat regression line for these dominance durations). This pattern of results was entirely characteristic of 5 of the 6 observers (G.H., K.Y., R.B., D.W., and H.W.).

For the 6th observer, dominance was not always restored by the flash; his nonperturbed dominance durations averaged $1.15 \mathrm{sec}$ during the perturbation period, which is less than $1 \mathrm{sec}$ briefer than his average unperturbed dominance durations. In all conditions-control trials included - this observer had very rapid rivalry alternations and reported difficulty tracking fluctuations in dominance. Because of the relative ineffectiveness of the perturbation flash, we have excluded this observer's data from our 

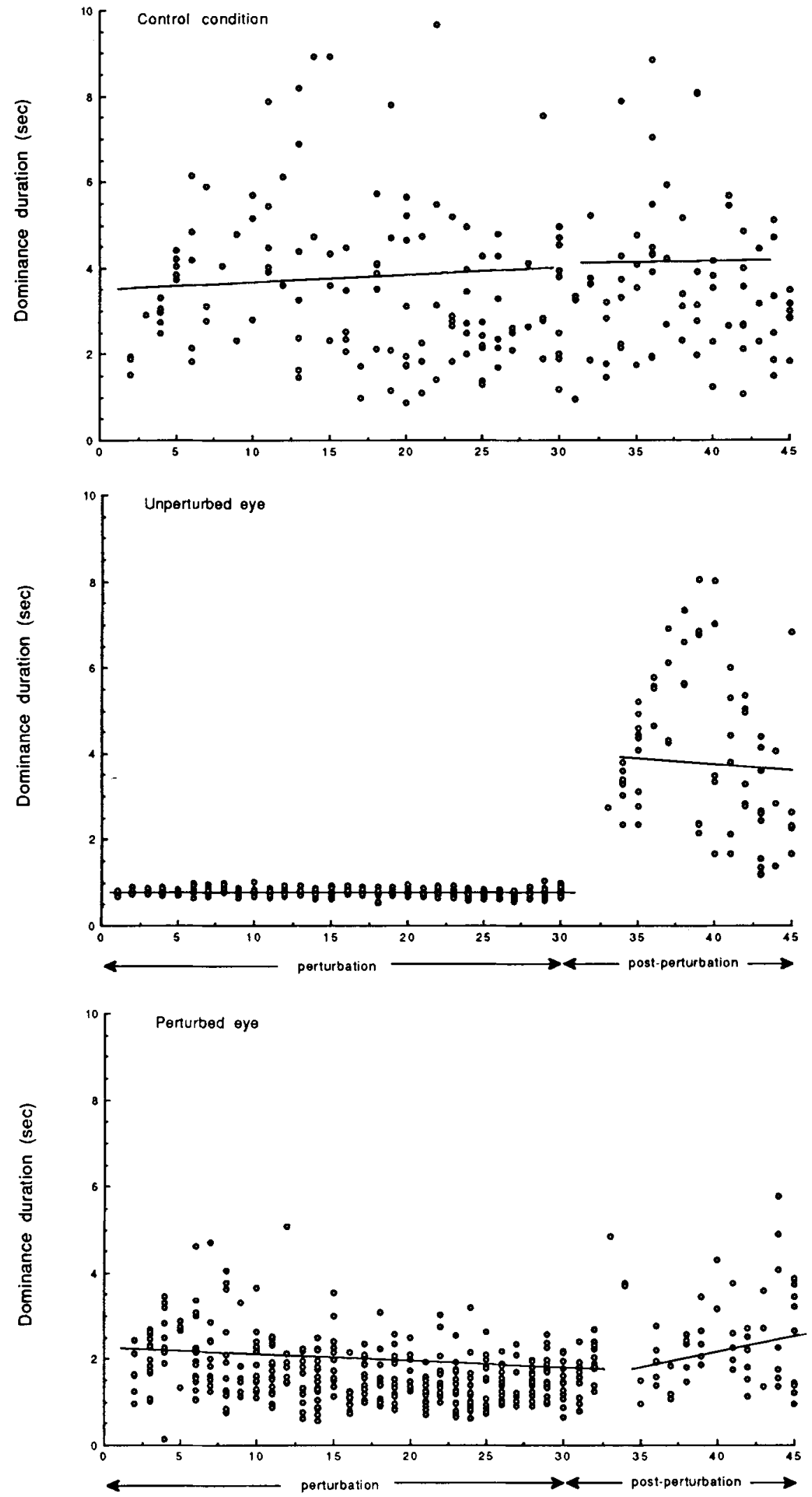

Time (sec) 
analyses. The remainder of this paper covers results and conclusions derived from the 5 observers for whom it was possible reliably to force dominance of one eye.

\section{Sequential Independence of Successive Dominance Phases}

Simple inspection of the successive dominance durations revealed no obvious trend for those durations to vary systematically throughout either the 30 -sec perturbation period or the 15 -sec postperturbation period. In the graphs in Figure 2, the absence of such a trend is evidenced by the wide scatter of dominance durations, the absence of periodicity in the plots, and the essentially flat regression lines. A specific test of sequential dependence was provided by an autocorrelation analysis, which has been used previously to study the stochastic properties of rivalry alternations (Blake et al., 1971; Fox \& Herrmann, 1967). For each observer's data, correlation coefficients were computed for the successive dominance durations associated with each eye; the number of lags tested depended on the number of sequential durations composing a tracking episode. For all lags, coefficient values were uniformly small and varied irregularly about zero. In brief, we detected no tendency for sequential dependence in these durations, for either the perturbation period or the postperturbation period.

\section{Average Dominance Durations \\ During Perturbation}

Having established that successive dominance durations remained independent even under conditions of perturbation, we next sought to determine whether the other temporal properties of rivalry alternations were affected. We computed mean dominance durations for each eye of each observer for both the perturbation period and the postperturbation period. Those averages are summarized in Figures 3 and 4 in the form of histograms.

Beginning with the results for the perturbation period, note first that the dominance durations for the nonperturbed eye are quite brief; this simply reflects the premature termination of suppression of the contralateral eye by perturbation of that eye's target. But note also that for observers R.B., G.H., K.Y., and H.W., the average dominance durations for the perturbed eye are themselves abbreviated, compared to dominance durations during the control sequence when no perturbations were introduced. This result is quite obvious in Figure 2; compare the dominance durations during perturbation in the lower graph (perturbed eye) to those in the upper graph (control condition). The difference between average dom- inance durations during control trials and those during perturbation trials is statistically significant for these 4 observers ( $p<.01$ for all comparisons). For these 4 individuals, in other words, an eye forced to return to dominance tends to revert to suppression sooner than normal. ${ }^{1}$

For the 5th observer (D.W.), the difference between control durations and perturbed durations was not statistically significant. However, D.W.'s rivalry alternation rate under control conditions was somewhat higher than that for the other 4 observers. This means, then, that dominance durations for D.W. were brief even during free-running rivalry, making it more difficult to detect abbreviated durations attributable to the perturbation. So we repeated the experiment on this observer at a much lower luminance level, a manipulation known to slow the rate of rivalry alternations (Levelt, 1965). For these measurements, the luminance of the white portions of the display was $1.0 \mathrm{fL}$ and the luminance of the black portions was unmeasurably low. Otherwise the procedures were the same as those employed in the first experiment.

Results obtained at this low luminance level are shown by the right-hand set of histograms in Figure 3 (DW-dim). Again perturbation successfully brought an eye back to dominance, as is evidenced by the consistently short dominance durations for the unperturbed eye. Moreover, autocorrelation analysis revealed no temporal dependencies in durations of successive phases of dominance. The lower luminance level did indeed slow rivalry alternations under the control conditions involving no perturbations. Moreover, the dominance durations for the perturbed eye were now consistently shorter than dominance durations under the control condition, consistent with the results of the other 4 observers. It appears, then, that the abbreviating effect of forced dominance of an eye occurs only if dominance durations exceed some minimum value. To the extent that individuals naturally differ in rate of rivalry alternations, the magnitude of the effect of forced dominance may vary from person to person.

\section{Average Dominance Durations \\ Following Perturbation}

Once the perturbation period ended, observers continued tracking rivalry for an additional $15 \mathrm{sec}$. As mentioned above, stochastic analyses of those successive phase durations revealed no significant dependencies; the temporal alternations continued to be random in nature. Figure 4 summarizes the average dominance durations for the perturbed and unperturbed eyes during this postperturbation period; there are no statistically significant

\footnotetext{
Figure 2 (opposite page). Scatterplot of individual dominance durations throughout the 45 -sec tracking period. In the top graph, neither eye's stimulus was perturbed at any time during the 45 -sec period; these data simply show the normal time course of rivalry alternations. In the middle and bottom graphs, the first 30 sec represent durations of dominance when one eye was forced to remain dominant, by briefly flashing (i.e., perturbing) the target viewed by that eye whenever the observer reported that it became suppressed. During the last $15 \mathrm{sec}$ (postperturbation), rivalry was allowed to free-run. The lower graph gives dominance durations for the eye receiving the perturbation (i.e., the eye forced to return to dominance), and the middle graph gives results for the eye not receiving the perturbation (i.e., the eye whose dominance was prematurely terminated). Included in the scatterplots are regression lines for these various conditions.
} 


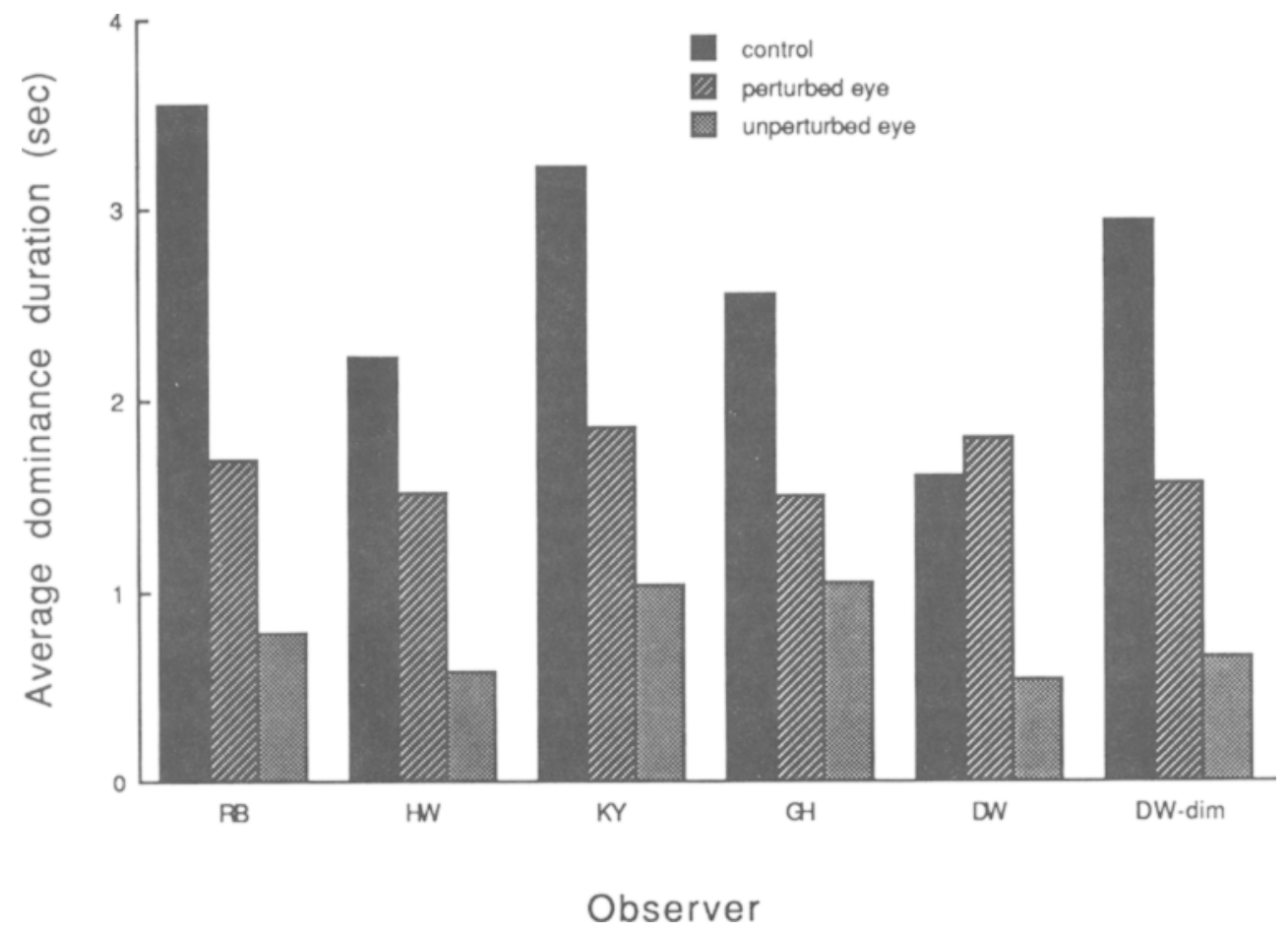

Figure 3. Histograms showing for each observer the average dominance durations during the 30-sec perturbation period.

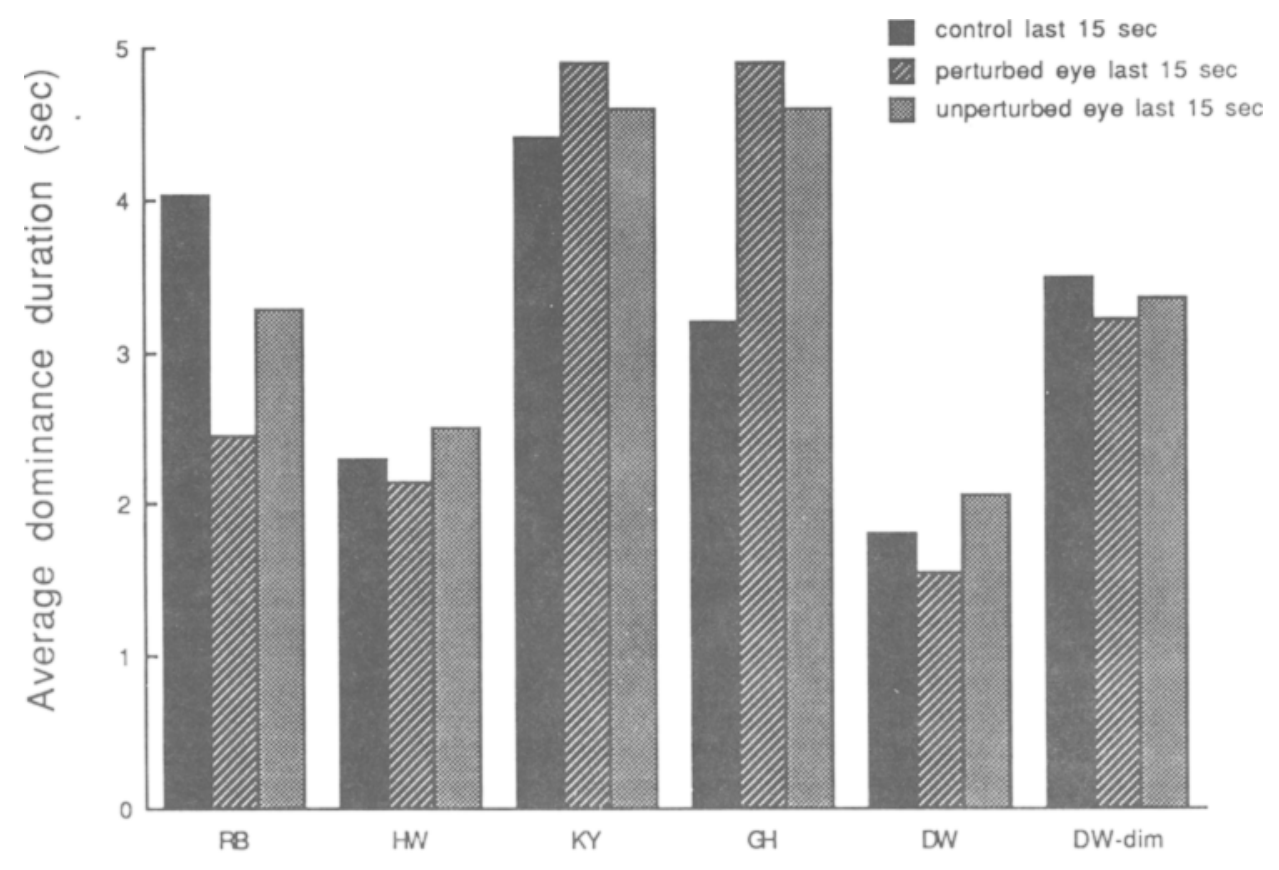

\section{Observer}

Figure 4. Histograms showing for each observer the average dominance durations throughout the 15-sec period immediately following the perturbation period. 


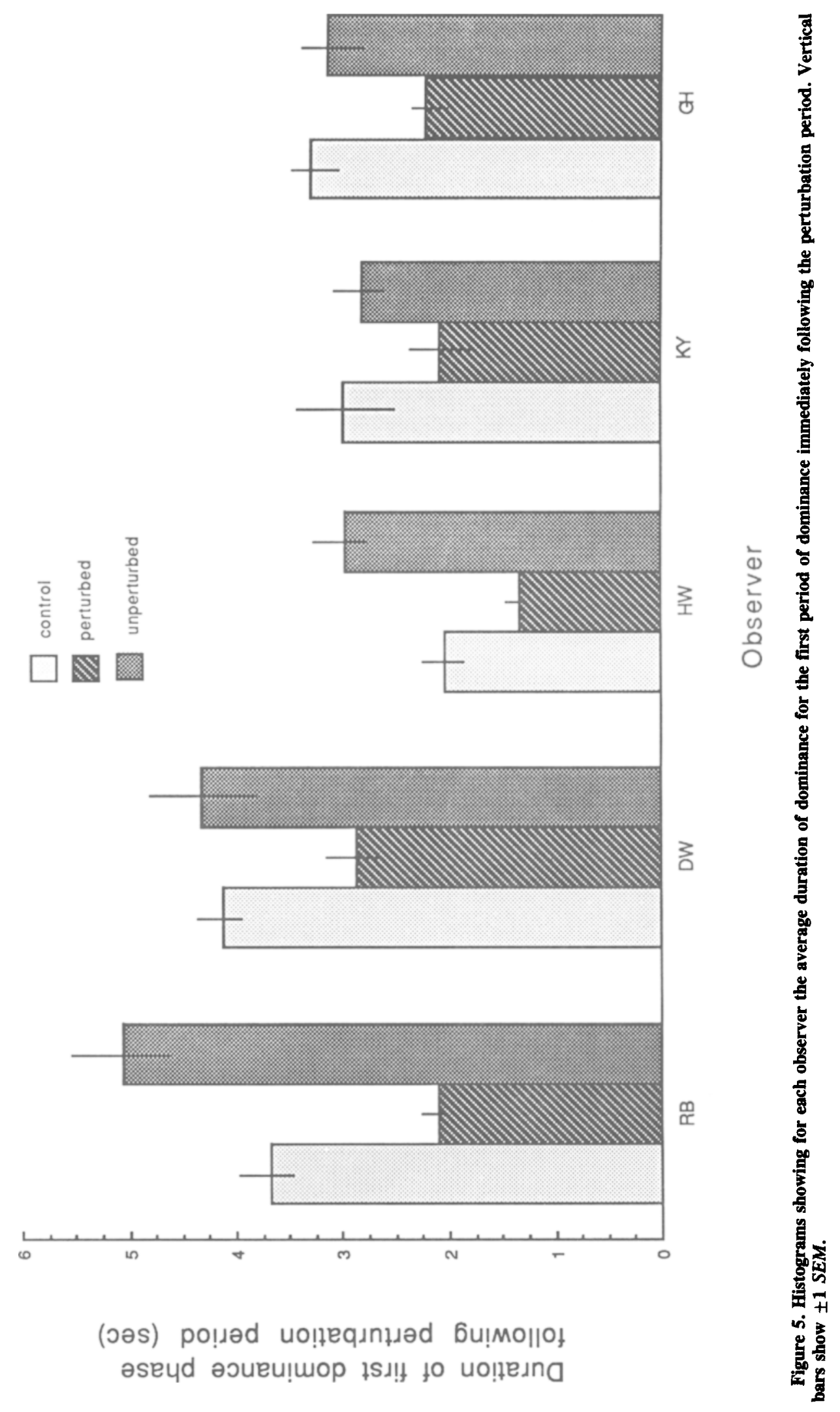


differences among durations for the previously perturbed eye, durations for the unperturbed eye, and control data. Inspection of the right-hand portions of the three graphs in Figure 2 reveal the same result. We next looked just at the first dominance duration immediately following the perturbation period, to see if those durations were unusual. As is shown by the histograms in Figure 5, the duration of the first dominance period for the previously perturbed eye was abnormally brief, whereas the first dominance duration for the unperturbed eye (i.e., the one forced to remain suppressed during the perturbation period) was abnormally long. There is, in other words, a significant carryover from the perturbation period. These trends are not seen, however, in durations for subsequent rivalry phases during the postperturbation period.

\section{Extended Period of Perturbation}

In the experiment just described, rivalry was perturbed for a 30-sec period. It is natural to wonder whether a more prolonged period of perturbation would have more drastic effects on the temporal course of rivalry. To find out, we retested two observers (R.B. and K.Y.), using a 240sec tracking episode divided into a 180 -sec perturbation period and a $60-\mathrm{sec}$ postperturbation test period. The same stimuli and procedures were employed. Since neither observer showed an eye asymmetry in the first experiment, we only tested with right-eye perturbation, repeating this extended sequence five times with rest periods interspersed.
Despite the considerably longer tracking/perturbation period, essentially the same pattern of results was obtained. Again there was no evidence for dependence of successive durations of dominance. Regression lines fitted to the successive durations (as in Figure 2) were essentially flat, indicating no tendency for durations to change systematically throughout the extended tracking episode, either during the perturbation period or during the postperturbation period. Shown in Figure 6 are the average dominance durations for the control condition, the perturbed eye, and the unperturbed eye, for both the period during perturbation (ON-the two sets of histograms in the left part of Figure 6) and the period immediately following perturbation (OFF-the two sets of histograms in the right part of Figure 6). The perturbation manipulation was effective throughout the 180 -sec period, as is evidenced by the very short dominance durations for the nonperturbed eye. Moreover, the perturbed eye dominance durations are significantly briefer than control dominance durations ( $p<.01$ for both observers). However, the average dominance durations during the postperturbation period (the right-hand set of histograms in Figure 6) are not significantly different. Finally, analysis of the first dominance duration following termination of the perturbation period disclosed the same result as that found in the earlier experiment: The first dominance duration for the previously perturbed eye averaged $1.30 \mathrm{sec}$ for R.B. and $2.10 \mathrm{sec}$ for K.Y.; compare these values to 4.27 and $4.02 \mathrm{sec}$, which are averages for

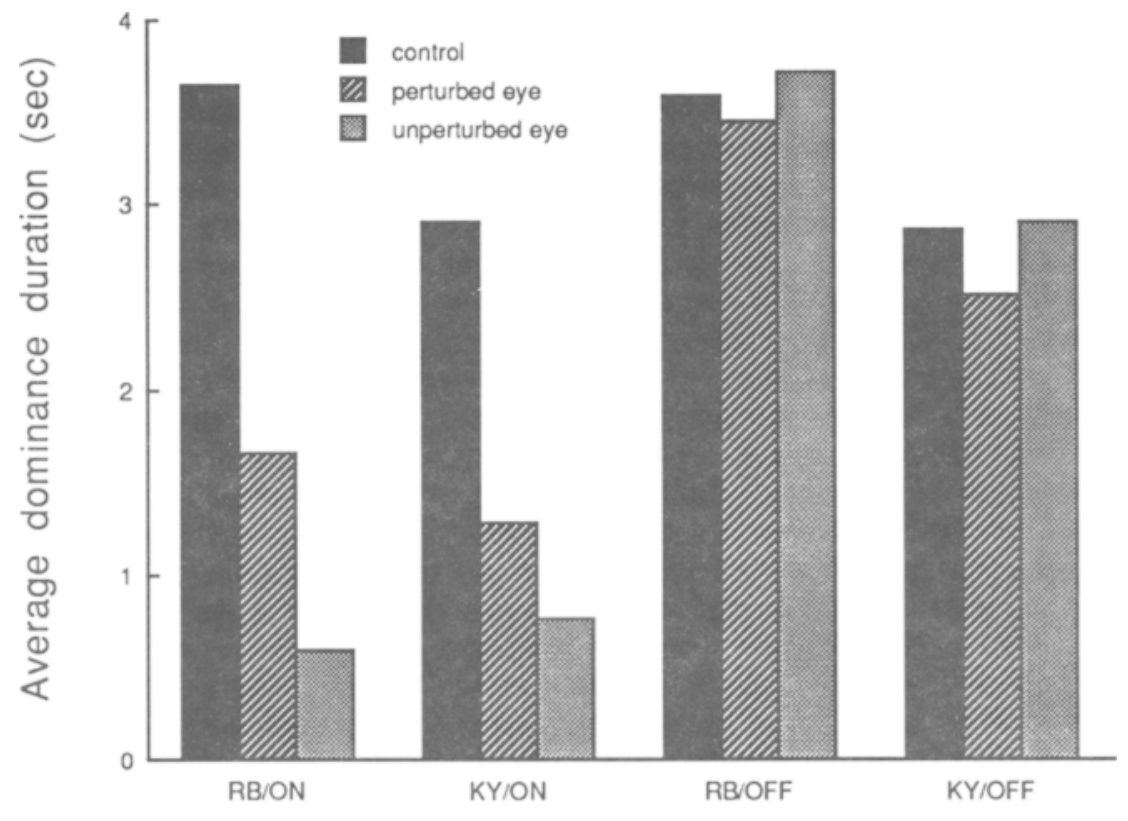

Observer/condition

Figure 6. Histograms showing the average duration of dominance during a 180-sec perturbation period (the two sets of histograms labeled $R B / O N$ and $K Y / O N$ ) and during a 60-sec period immediately following perturbation (RB/OFF and KY/OFF). 
the first dominance duration under the control condition. Once again, then, an eye, immediately upon being released from forced dominance, generates an unusually brief dominance duration. There is no indication, however, that this reactionary abbreviation in dominance is any greater with long $(180-\mathrm{sec})$ rather than short (30sec) episodes of forced dominance, and the recovery (i.e., return to normal free-running rivalry) seems to occur just about as quickly.

\section{Demonstration of the Perturbation Effect}

The effect of perturbation described in this paper is compelling and can be easily experienced by readers who view stereoscopically the rival targets shown in Figure 1 (or any other dissimilar monocular targets that instigate rivalry). Take a few moments to get an impression of the length of the dominance durations of, say, the white disk. Now move a pencil or the index finger in front of the eye viewing that target every time it becomes suppressed. This action, by creating a transient in the suppressed eye, should return that stimulus to dominance. Now, repeating this "perturbation" each time the disk becomes suppressed, pay attention to the durations of dominance of the disk. You should notice that the disk remains dominant for only short periods of time. If you find the rate of rivalry alternations quite rapid, try viewing the rival targets through a pair of sunglasses or under reduced illumination.

\section{DISCUSSION}

The present results are generally relevant for models of rivalry incorporating the notions of reciprocal inhibition and adaptation. A key assumption in these models involves some version of the concept of reciprocal inhibition, as developed originally by Sherrington (1906) to characterize the continual interplay of excitation and inhibition within spinal cord neurons mediating flexion and extension of muscles. Impressed by the power of this concept, William McDougall (1906), a contemporary of Sherrington, applied reciprocal inhibition as a general explanatory mechanism for multistable perceptual phenomena, including binocular rivalry. In this application, the endpoints are now percepts rather than muscular responses. Reciprocal inhibition has continued to occupy a prominent position in contemporary models of the rivalry alternation process (Lehky, 1988; Matsuoka, 1984; Mueller, 1990; Sugie, 1982). In these contemporary models, temporal variations in excitation and inhibition account for changes in dominance and suppression-a dominant eye becomes suppressed because the excitation supporting dominance is weakened through adaptation, or fatigue, and recovery from adaptation occurs during the suppression phase. ${ }^{2}$

Now, with these general notions in mind, consider what should happen when an eye is forced to remain dominant, as in our experiments. Because perturbation prevents the excitatory and inhibitory processes from completing their normal course, the interplay between them, which is the essence of reciprocity, is unbalanced. Consequently, a surplus of some excitatory and inhibitory quantity will remain and participate in the next rivalry cycle. Moreover, it is reasonable to assume that this remainder will accrue as perturbation continues, leading to a departure from sequential independence. In fact, however, such a departure was not observed for either the 30 - or the $180-\mathrm{sec}$ perturbation period. In both conditions, sequential independence was maintained. Results from the 180 -sec perturbation period are especially noteworthy, because an adaptation period of this duration is of sufficient magnitude to induce many kinds of perceptual aftereffects usually attributed to fatigue-like processes.

Although the absence of sequential dependency does not fit well with the assumptions of reciprocal inhibition, the abbreviated dominance durations observed during perturbation are generally consistent with those assumptions. Reciprocal inhibition models (e.g., Lehky, 1988) posit that the eye being denied suppression has no opportunity to recover from adaptation; hence its excitatory strength becomes abnormally depleted while the excitatory strength of its partner eye (the one forced to remain suppressed) becomes abnormally high. Hence, as we found, the dominance durations of an eye forced to remain dominant are unusually brief. Even so, the absolute magnitude of these reductions in dominance durations are small, on the order of $1 \mathrm{sec}$. Although we have no principled basis for defining "small,"' it is noteworthy that these foreshortened dominance durations fall within the range of durations found during the control period. Moreover, the durations found for the first rivalry cycle immediately following the perturbation period are also congruent with the concept of reciprocal inhibition. Specifically, the dominance duration increased for the eye previously forced to be suppressed and decreased for the eye forced to be dominant. It is interesting that these transient changes in dominance durations endure for only one cycle, after which rivalry returns to free-running levels.

The only way to determine if the magnitude and brevity of this perturbation effect comport with the key assumption of reciprocal inhibition is to use quantitative analysis. Fortunately, the existence of several models (Lehky, 1988; Matsuoka, 1984; Mueller, 1990; Sugie, 1982) render such an analysis feasible. Indeed, the capacity to simulate our results, while retaining reasonable assumptions, can serve as a test of the viability of specific models. More generally, simulations can address the question of the suitability of the concept of reciprocal inhibition as a theoretical framework for encompassing rivalry.

Apart from theoretical issues, the remarkable empirical aspect of our data is the modest impact that perturbation exerted on the temporal pattern of rivalry. Sequential independence of successive durations was maintained under all conditions, and, after only one cycle, the temporal pattern returned to nonperturbated values. To us, this implies that the mechanism underlying rivalry possesses considerable autonomy. 


\section{REFERENCES}

Blake, R., Fox, R., \& McIntyre, C. (1971). Stochastic properties of stabilized-image binocular rivalry alternations. Journal of Experimental Psychology, 88, 327-332.

Fox, R., \& HerrmanN, J. (1967). Stochastic properties of binocular rivalry alternations. Perception \& Psychophysics, 2, 432-436.

Fox, R., Todd, S., \& BetTinger, L. A. (1975). Optokinetic nystagmus as an objective indicator of binocular rivalry. Vision Research, 15, 849-853.

LACK, L. (1978). Selective attention and the control of binocular rivalry. The Hague: Mouton.

LEHKY, S. (1988). An astable multivibrator model of binocular rivalry. Perception, 17, 215-228.

Levelt, W. J. M. (1965). On binocular rivalry. Soesterberg, The Netherlands: Institute for Perception RVO-TNO.

Matsuoka, K. (1984). The dynamic model of binocular rivalry. Biological Cybernetics, 49, 201-208.

MCDougall, W. (1906). The physiological factors of the attention process IV. Mind, 15, 329-359.

MUELLER, T. J. (1990). A physiological model of binocular rivalry. Visual Neuroscience, 4, 63-74.

SHERRINGTON, C. S. (1906). The integrative action of the nervous system. New Haven, CT: Yale University Press.

SuGIE, N. (1982). Neural models of brightness perception and retinal rivalry in binocular vision. Biological Cybernetics, 43, 13-21.

WADE, N. J. (1975). Binocular rivalry between single lines viewed as real images and afterimages. Perception \& Psychophysics, 17, 571-577.

WALKER, P. (1975). Stochastic properties of binocular rivalry alternations. Perception \& Psychophysics, 18, 467-473.

WoLFE, J. M. (1984). Reversing ocular dominance and suppression in a single flash. Vision Research, 24, 471-478.

\section{NOTES}

1. One referee worried whether the brief perturbation flashes themselves were responsible for reducing the average dominance durations of an eye, independent of the phenomenal state of the eye receiving the flashes. At the suggestion of that referee, we tested 1 individual (R.B.) under conditions in which the flash was presented during the dominance phase of rivalry rather than when the eye was suppressed. Brief transients during dominance had no influence on the temporal course of rivalry nor on the average dominance durations for that eye or the eye not receiving transient stimulation. The effectiveness of perturbation flashes is related to the premature termination of suppression.

2 . The randomness of successive durations is not an inherent property of reciprocal inhibition. Indeed, recent reciprocal inhibition models of rivalry have simply incorporated a noise component to reconcile their predictions with actual rivalry data.

(Manuscript received March 30, 1990; revision accepted for publication July 20, 1990.) 